\title{
Medical abortion in primary care
}

\section{SUMMARY}

Early medical abortion is a safe, cost effective and acceptable alternative to surgical abortion. It offers privacy and autonomy and can be provided by general practitioners who have completed an online training module.

Abortion can be induced with mifepristone and misoprostol up to nine weeks of gestation. Apart from anticoagulation and severe anaemia, there are few contraindications and precautions for medical abortion.

Obtaining informed consent requires the provision of information on expected effects, adverse effects and complications. The woman must know when to present to an emergency department.

Follow-up must be arranged to ensure the abortion is complete. A serum human chorionic gonadotropin concentration or a low-sensitivity urine pregnancy test is used to confirm completion.

Most contraceptive methods can be started immediately following medical abortion. If an intrauterine device is preferred, it should be inserted after confirming the abortion is complete.

\section{Introduction}

In Australia, approximately $25 \%$ of pregnancies are unplanned, of which one-third end in abortion. Early medical abortion is an alternative to surgical abortion for pregnancies up to nine weeks gestation. It is generally less costly than surgical abortion and the drugs used (mifepristone and misoprostol) are subsidised by the Pharmaceutical Benefits Scheme. Early medical abortion offers privacy and autonomy. It can be supported by telemedicine and generally avoids the need for invasive surgical procedures.' Clinical advice in this article is based on the medical abortion section of Therapeutic Guidelines: Sexual and Reproductive Health. ${ }^{2}$

\section{Medical abortion in primary care}

Currently only around 2850 of the 41,000 GPs in Australia are registered to prescribe the mifepristone and misoprostol regimen for medical abortion. To register they must complete the free 3-4 hours online training of the MS-2 Step Prescribing Program. This is mandatory except for those who hold a current Fellowship or Advanced Diploma of the Royal Australian and New Zealand College of Obstetricians and Gynaecologists.

While women need advice about what early medical abortion involves, the majority do not require counselling about their decision to have an abortion, although some will require additional support. A Medicare rebate is available for up to three nondirective counselling sessions on pregnancy options delivered by an endorsed GP, psychologist, social worker or mental health nurse. For women without contraindications to either medical or surgical abortion the relative advantages and disadvantages of both methods should be discussed (Box 1). When feasible, the chosen method should be offered or facilitated.

Contraceptive options are important to raise before an abortion. This includes discussing long-acting reversible contraceptive methods. Support for clinicians providing, or considering providing, medical abortion in primary care is available through the Australian Contraception and Abortion Primary Care Practitioner Support Network (AusCAPPS).

\section{Contraindications}

There are few contraindications to medical abortion for women with a confirmed intrauterine pregnancy of no more than 63 days. Important contraindications are shown in Box 2. Breastfeeding and multi-fetal pregnancies are not contraindications. Early medical abortion is clinically preferred over surgical abortion if there are anaesthetic risks and may be safer for obese women and those with distortion of the uterine cavity due, for example, to large fibroids.

\section{Precautions}

Specialist advice is required for women with severe or respiratory disease. Due to the antiglucocorticoid effects of mifepristone, specialist advice is needed for those with asthma that is difficult to control. This is because there may be no effect if the patient needs anaemia, ischaemic heart disease and severe renal

\section{Deborah Bateson}

Medical director, Family Planning New South Wales, Ashfield, Sydney

Clinical associate professor, Specialty of Obstetrics, Gynaecology and Neonatology, Faculty of Medicine and Health, University of Sydney

\section{Kathleen McNamee (D)}

Medical director, Family Planning Victoria, Box Hill, Melbourne

Senior lecturer, Department of Obstetrics and Gyneacology, Monash University, Melbourne

\section{Caroline Harvey (D)}

Senior general practitioner, Sexual and Reproductive Health, Institute for Urban Indigenous Health, Brisbane

\section{Keywords}

induced abortion, mifepristone, misoprostol

Aust Prescr 2021;44:187-92 https://doi.org/10.18773/ austprescr.2021.050 
to increase the dose of the corticosteroid she uses for asthma control. For those with stable asthma on long-term inhaled preventer therapy, review the asthma plan to ensure appropriate steps can be taken in case of an exacerbation. Careful glucose monitoring may be needed for women using insulin because of possible vomiting, altered food and liquid intake, and potential dehydration during the abortion. Additional care is also required for women with epilepsy who have seizures induced by vomiting or pain.

\section{Box 1 Relative advantages of medical and surgical abortion ${ }^{2}$}

\section{Medical abortion}

Usually avoids invasive procedures and potential surgical complications (e.g. uterine perforation, anaesthetic risk)*

May be safer for individuals with obesity or distortion of the uterine cavity

May be more widely accessible

Usually less costly

Usually allows abortion to take place at home

Seen by some individuals as a more natural and less medical process

\section{Surgical abortion}

Less likely to require subsequent evacuation of retained products*

Requires only one appointment and is usually performed under sedation

Causes less pain

Bleeding resolves in a few days rather than weeks

Less risk of severe bleeding and access to emergency care not usually required

Avoids potential distress of seeing the gestational sac

* In 3-5\% of medical abortions and less than $1 \%$ of surgical abortions, subsequent surgical evacuation of retained products of conception is required.

\section{Box 2 Contraindications and precautions in medical abortion}

Contraindication to mifepristone and misoprostol combination

Intrauterine device (IUD) in place - if the IUD cannot be removed, a surgical abortion is the recommended safe option

Haemorrhagic disorder or treatment with anticoagulants

Long-term use of an oral corticosteroid (effectiveness may be reduced by the antiglucocorticoid action of mifepristone)

A travel time to hospital emergency services with blood transfusion services of more than two hours in the 14 days after taking mifepristone

Confirmed or suspected ectopic pregnancy

Hypersensitivity to mifepristone, misoprostol (or any prostaglandin)

Uncertainty about gestational age

Inherited porphyria - there is a theoretical risk of precipitating or exacerbating attacks of porphyria, but no data are available

Precautions for mifepristone and misoprostol combination

Severe anaemia

Ischaemic heart disease

Asthma that is unstable or treated with long-term preventer therapy

Insulin requiring diabetes

Epilepsy

\section{Investigations}

Before a medical abortion it is important to confirm that there is an intrauterine pregnancy. The mifepristone and misoprostol combination is not effective for ectopic pregnancy.

\section{Ultrasound}

An intrauterine pregnancy is confirmed by the presence of either a fetal pole or yolk sac. These structures are usually visible at 5-6 weeks gestation on a high-quality vaginal ultrasound. Confirmation may be delayed if transabdominal ultrasound is used, particularly in obese women. ${ }^{2}$

\section{Human chorionic gonadotropin}

Measurement of the quantitative serum human chorionic gonadotropin (HCG) concentration is recommended on the day (or as soon as possible before) mifepristone is taken. This baseline value enables comparison with a repeat measurement seven days after the dose of mifepristone, to confirm completion of the abortion.

For pregnancies that are suspected to be less than six weeks, the serum HCG can be useful to guide the timing of the ultrasound. This reduces the need for a repeat scan.

If the HCG is more than $5400 \mathrm{IU} / \mathrm{L}$, there is a $90 \%$ chance a fetal pole or yolk sac will be detected on a high-quality vaginal ultrasound. At lower concentrations ultrasound can be delayed, unless there is suspicion of ectopic pregnancy.

\section{Other investigations}

Investigations to be considered include:

- $\quad$ screening for sexually transmitted infections (chlamydia, gonorrhoea)

- haemoglobin and iron studies if anaemia is known or suspected, for example in women with heavy menstruation or from populations with a high prevalence of iron deficiency.

\section{Procedure}

The woman must give informed consent for the procedure. This includes provision of information about how to take the drugs, their expected effects, adverse effects and possible complications, including advice on when to present to an emergency department.

* A clinical trial is currently underway in Australia of very early medical abortion (VEMA) before an intrauterine pregnancy can be confirmed. VEMA is not yet mainstream practice. 
The regimen for medical abortion is supplied in a composite pack containing one mifepristone $200 \mathrm{mg}$ tablet and four misoprostol 200 microgram tablets. Mifepristone blocks the action of progesterone which supports the endometrium in a continuing pregnancy. It also softens and dilates the cervix, increases uterine activity and increases sensitivity to misoprostol. Misoprostol is a synthetic prostaglandin $E_{1}$ analogue which softens the cervix and induces uterine contractions to expel the pregnancy.

Step 1: Mifepristone is taken orally. Adverse effects are uncommon, but if vomiting occurs within one hour of ingestion, the dose must be repeated. Normal activities can be continued. Light bleeding is common. The pregnancy is expelled in only around $5 \%$ of cases before taking misoprostol, however it is important the misoprostol is taken as planned to reduce the chances of retained products.

Step 2: It is recommended that pre-medication with an antiemetic and a non-steroidal anti-inflammatory drug is taken 30-60 minutes before misoprostol and that a small quantity of stronger analgesics is supplied for use if needed. Stronger analgesics may be taken before misoprostol to manage pain. Some women choose to use these only if necessary. Misoprostol is taken buccally 36-48 hours after mifepristone at a pre-arranged time to suit the patient's schedule. The mouth is rinsed with water and two tablets are placed on each side between the lower gums and cheeks and held in place for 30 minutes. Any residual material is then swallowed. Misoprostol can cause adverse effects including nausea, headaches, fever and diarrhoea. ${ }^{3-5}$

\section{Expected effects}

Cramping pain, generally worse than menstrual pain, can start within 1-4 hours of taking misoprostol. Bleeding generally follows pain and is usually heavier than normal menstruation and blood clots are usual. This bleeding generally settles after the products of conception are passed. The conceptus is more likely to be seen with later gestations. This is distressing for some women and reassuring for others. Bleeding remains similar to menstruation for 5-7 days before gradually decreasing. Fluctuations in the amount of bleeding and clots are common. Bleeding stops by day 14 in approximately $65 \%$ of cases, but light bleeding or spotting can persist for 4-5 weeks.

\section{Follow-up}

All women should be followed up after early medical abortion. Printable information for patients is available from Therapeutic Guidelines: Sexual and Reproductive Health (Fig). ${ }^{2}$ This includes advice on avoiding intercourse, tampons, baths and swimming for seven days to reduce the risk of infection. There is guidance on when to present to an emergency department in the event of heavy bleeding or severe pain.

Anti-D prophylaxis for Rhesus-negative patients is no longer required for early medical abortion up to 10 weeks gestation. ${ }^{6}$

\section{Check-in call at three days}

Most practitioners schedule a routine call with the patient around three days after mifepristone is taken to check whether the bleeding was as expected. This is also to check for possible complications associated with ongoing pain, heavier than expected bleeding or ongoing symptoms of pregnancy.

There are two methods to confirm there is no ongoing viable pregnancy. The most common method is to measure the serum HCG concentration. There should be an $80 \%$ or greater fall from the baseline test to a follow-up test seven days after taking mifepristone. Alternatively, a low-sensitivity urine pregnancy test can be supplied for home use 16-21 days after the mifepristone is taken. If the test is positive, the serum HCG must be measured to exclude ongoing pregnancy. Standard urine pregnancy tests are unsuitable. They are sensitive to $25 \mathrm{IU} / \mathrm{L}$ so are likely to remain positive for several weeks after a medical abortion. Low-sensitivity tests only detect HCG concentrations over $1000 \mathrm{IU} / \mathrm{L}$. Routine use of ultrasound to assess the outcome is not recommended.

\section{Follow-up at 2-3 weeks}

The 2-3-week follow-up can be in person or by telehealth methods. The aim is to check that the abortion has been successful with no ongoing viable pregnancy, that there are no symptoms suggestive of complications and to ensure contraceptive needs are met.

A range of emotions may be experienced before and after an abortion including grief and sadness, but for most women these are transient and often accompanied by relief. Some people will experience distress and may require extra support and referral to a mental health practitioner. Additional care may be required for women with a pre-existing mental health disorder. There is no evidence that abortion is associated with an increased risk of adverse mental health outcomes compared to continuing with an unplanned pregnancy. ${ }^{5,7}$

\section{Contraception after medical abortion}

Another pregnancy is possible soon after an abortion. With the exception of an IUD, all contraceptive methods can be started soon after taking mifepristone (Table). There is a theoretical concern that progestogen-only methods, started before taking mifepristone, could 


\section{Fig. Advice after a medical abortion}

\section{Therapeütic Guidelines}

\section{Advice after a medical abortion}

\section{General advice after a medical abortion}

A follow-up blood test is very important to check that the abortion has been successful. The test needs to be taken 7 days after you took the first tablet (mifepristone). Other forms of testing may be appropriate for some people, but your clinic will discuss these with you if they are an option.

For 7 days after taking the second tablet (misoprostol), to reduce the risk of infection, avoid:

- sexual intercourse

- use of tampons or menstrual cups

- swimming

- taking a bath or using a spa.

When to go to an emergency department

Go to an emergency department if at any time you have:

- very heavy bleeding, such as any of the following:

- your bleeding fills more than two large pads in an hour for more than 2 hours in a row

- you are passing clots the size of a small lemon or larger

- you feel faint and think the bleeding is heavy even if you are not sure about how much you are bleeding

- any of the following symptoms (which could mean an ectopic pregnancy in the Fallopian tube):

- severe abdominal (tummy) pain

- pain in your pelvis on one side

- pain in the tips of your shoulders

- other concerns and you don't have access to medical advice (eg from the prescribing clinic).

When to contact the clinic that prescribed the abortion drugs

If you have any of the symptoms below, you might still be pregnant. Contact the clinic if:

- at $\mathbf{2 4}$ hours after taking misoprostol, you either:

- have had no or little bleeding (less than a normal period), or

- have not passed any pregnancy tissue, or any clots larger than a small grape

- at $\mathbf{4 8}$ hours after taking misoprostol, you still have nausea

- you had some initial bleeding, but it stopped within 4 days of taking misoprostol

- at 14 days after taking misoprostol, you still have breast tenderness.

If you have any of the symptoms below, there might still be some pregnancy tissue (eg placenta) in the uterus (womb) Contact the clinic if:

- at 7 days after taking misoprostol:

- you are still passing clots

- you still have cramping pain

- you still have bleeding that is heavier than a period

- you have bleeding that stopped and restarted and has been as heavy as a period for the last 24 hours or more

- at 14 days after taking misoprostol you have bleeding that is not much less than when it started

- at 4 to 5 weeks after taking misoprostol you still have bleeding that is different to your usual menstrual cycle.

Contact the clinic if you have any of the symptoms below, as they can indicate that you have an infection of the uterus:

- pelvic pain

- pain during sex

- unusual vaginal discharge

- fever (over $38^{\circ} \mathrm{C}$ )

- tenderness on touching the abdomen (tummy) or pelvis

- nausea or vomiting

- feel unwell.

Contact the clinic that prescribed the abortion drugs if you have any concerns about the medical abortion.

Doctor's contact details:

Therapeutic Guidelines Limited (www.tg.org.au) is an independent not-for-profit organisation dedicated to deriving guidelines for therapy from the latest world literature, interpreted and distilled by Australia's most eminent and respected experts. 
reduce the effectiveness of mifepristone. While evidence is limited, those choosing a depot injection should be advised of this potential effect. A number of studies have shown the contraceptive implant does not affect the efficacy of mifepristone. ${ }^{4}$

\section{Complications}

Complications of early medical abortion are uncommon, but important to recognise and manage appropriately. The complication rates from international and national data are:

- retained products of conception requiring surgical intervention - up to $5 \%$

- continuing viable pregnancy $-0.8 \%$

- upper genital tract infection requiring intravenous antibiotics - 0.1-0.2\%

- haemorrhage requiring transfusion $-0.1 \%{ }^{8}$

The advice given to women on the symptoms that warrant going to an emergency department can be supported by the printable patient information sheet from Therapeutic Guidelines (Fig.).

\section{Retained products of conception}

The retention of tissue after passage of the conceptus is suspected by ongoing heavier than expected bleeding and cramping. Retained products can be confirmed by ultrasound, but this is not always necessary if the person is well and the bleeding is settling.

Management depends on symptoms such as painful cramping, the size of the retained products, the development of anaemia and the patient's preferences. The options are expectant management, or repeat dosing of misoprostol (400 microgram buccally, with an additional 400 microgram buccally in four hours if no significant bleeding has occurred), or surgical curettage. Antibiotics are required if infection is suspected.

\section{Continuing pregnancy}

An ongoing viable pregnancy should be suspected if little, light or no bleeding occurred within 24 hours of taking misoprostol, particularly if products of conception were neither seen nor felt to have passed and pregnancy symptoms are continuing. An ultrasound and quantitative HCG are generally required. If pregnancy is confirmed and the gestation remains within 63 days, mifepristone and misoprostol can be repeated, otherwise a surgical procedure is required. If the woman wants to continue with the pregnancy, she must be informed that there may be a risk of significant fetal abnormality. Referral for specialist management of the pregnancy is recommended.

\section{Table Medical abortion and starting contraception}

\begin{tabular}{|c|c|c|}
\hline $\begin{array}{l}\text { Contraceptive } \\
\text { method }\end{array}$ & Timing of initiation & $\begin{array}{l}\text { Time to } \\
\text { effectiveness }\end{array}$ \\
\hline \multirow{2}{*}{$\begin{array}{l}\text { Etonogestrel implant } \\
\text { Medroxyprogesterone } \\
\text { acetate injection }\end{array}$} & $\begin{array}{l}\text { On the day of or within } 5 \text { days of } \\
\text { taking mifepristone }\end{array}$ & Immediately \\
\hline & $\begin{array}{l}\text { More than } 5 \text { days post } \\
\text { mifepristone* }\end{array}$ & 7 days \\
\hline \multirow[t]{3}{*}{ Intrauterine devices } & Exclude new pregnancy & \multirow{3}{*}{$\begin{array}{l}\text { Copper IUD: } \\
\text { immediately } \\
\text { Hormonal IUD: } 7 \text { days } \\
\text { or immediately if day } \\
1-5 \text { of menstrual cycle }\end{array}$} \\
\hline & $\begin{array}{l}\text { Insert after completion of abortion, } \\
\text { provided no symptoms of retained } \\
\text { products, or at the time of the first } \\
\text { menstrual period }\end{array}$ & \\
\hline & $\begin{array}{l}\text { Encourage hormonal bridging } \\
\text { method until insertion }\end{array}$ & \\
\hline \multirow{2}{*}{$\begin{array}{l}\text { Combined hormonal } \\
\text { contraception }{ }^{\dagger} \text { and } \\
\text { progestogen-only pill }\end{array}$} & Within 5 days of taking mifepristone & Immediately \\
\hline & More than 5 days post mifepristone* & 7 days $\ddagger$ \\
\hline
\end{tabular}

IUD intrauterine device

* Exclude new pregnancy. A follow-up pregnancy test is required if pregnancy is not excluded

+ Vaginal ring inserted after the heaviest bleeding has decreased

$\ddagger$ Three consecutive pills (over 48 hours) for norethisterone 350 microgram and levonorgestrel progestogen-only pill.

\section{Upper genital tract infection}

Infection can present with subtle symptoms. Most cases are associated with retained products of conception. Upper genital tract infections are polymicrobial, most commonly involving anaerobic vaginal bacteria. Severe infections can be caused by Clostridium species or Streptococcus pyogenes. Sexually transmitted infections Chlamydia trachomatis, Neisseria gonorrhoea and Mycoplasma genitalium must also be considered.

Treat suspected endometritis with oral amoxicillin in combination with clavulanic acid for seven days (private script or streamlined authority is required for courses longer than five days). Severe infection will require inpatient intravenous antibiotics. Consider sexually transmissible infections in at-risk unscreened patients.

\section{Conclusion}

Early medical abortion in primary care offers an alternative safe and effective choice to surgical abortion. General practitioners are ideally placed to support their patients' reproductive health and autonomy by providing medical abortion and follow-up care. $<$

Conflicts of interest: none declared 
<ustralian Prescriber

\section{REFERENCES}

1. Deb S, Subasinghe AK, Mazza D. Providing medical abortion in general practice: General practitioner insights and tips for future providers. Aust J Gen Pract 2020;49:331-7. https://doi.org/10.31128/AJGP-01-20-5198

2. Medical abortion. In: Therapeutic Guidelines [digital] Melbourne: Therapeutic Guidelines Limited; 2021 https://www.tg.org.au [cited 2021 Sep 21]

3. Royal Australian and New Zealand College of Obstetricians and Gynaecologists. The use of mifepristone for medical abortion. Melbourne: RANZCOG; 2019. https://ranzcog.edu.au/ RANZCOG SITE/media/RANZCOG-MEDIA/Women\%27s\% $20 \mathrm{Health} /$ Statement\%20and\%20guidelines/Clinical\%20 -\%20Gynaecology/Use-of-mifepristone-for-medical-abortion (C-Gyn-21)Review-March-2019 2.pdf?ext=.pdf [cited 2021 Sep 21]

4. National Institute for Health and Care Excellence. Abortion care. London: NICE; 2021. https://www.nice.org.uk/ guidance/ng140 [cited 2021 Sep 21]
5. Royal College of Obstetricians and Gynaecologists. Best practice in comprehensive abortion care. Best Practice Paper No. 2. London: RCOG; 2015. https://www.rcog.org.uk/ en/guidelines-research-services/guidelines/bpp2 [cited 2021 Sep 21]

6. National Blood Authority. Prophylactic use of Rh D immunoglobulin in pregnancy care. Canberra: National Blood Authority; 2021. https://www.blood.gov.au/anti-d-0 [cited 2021 Jun 9]

7. Steinberg JR. Decision rightness and relief predominate over the years following an abortion. Soc Sci Med 2020;248:112782. https://doi.org/10.1016/j.socscimed.2020.112782

8. Goldstone P, Walker C, Hawtin K. Efficacy and safety of mifepristone-buccal misoprostol for early medical abortion in an Australian clinical setting. Aust N Z J Obstet Gynaecol 2017:57:366-71. https://doi.org/10.1111/ajo.12608 